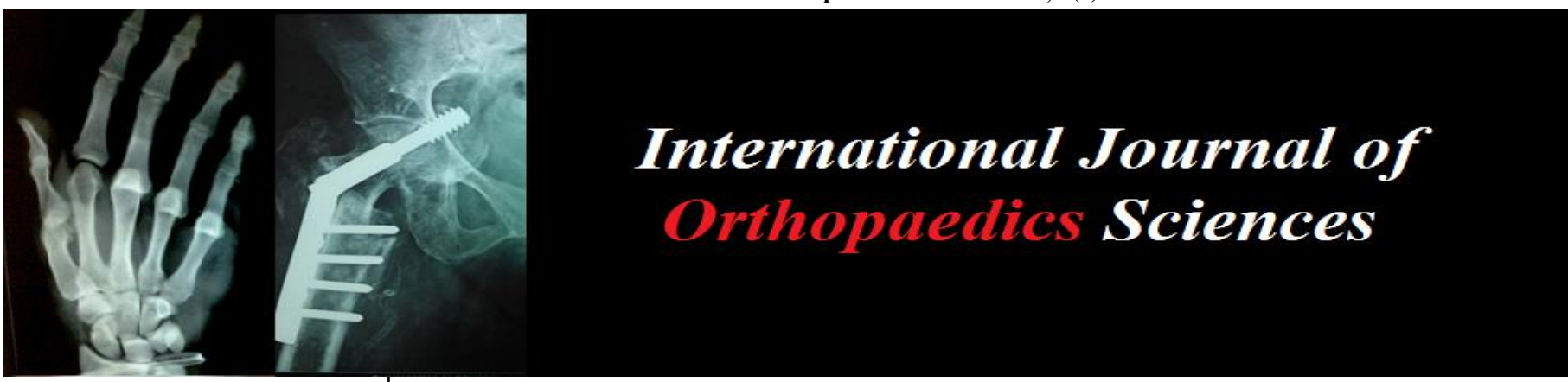

ISSN: $2395-1958$

IJOS 2019; 5(2): 1028-1031

(C) 2019 IJOS

www.orthopaper.com

Received: 24-02-2019

Accepted: 28-03-2019

Dr. Subraya Bhat Kuloor

Associate Professor, Department of Orthopaedics, Govt Medical

College Kannur, Pariyaram,

Kerala, India

Dr. Abdul Jameel Shareef Professor, Department of Orthopaedics, Govt Medical College Kannur, Pariyaram, Kerala, India
Correspondence

Dr. Subraya Bhat Kuloor Associate Professor, Department of Orthopaedics, Govt Medical College Kannur, Pariyaram,

Kerala, India

\section{Suturing and wiring a novel technique for Osteosynthesis of inferior pole patella fracture: A prospective comparative study}

\section{Dr. Subraya Bhat Kuloor and Dr. Abdul Jameel Shareef}

DOI: https://doi.org/10.22271/ortho.2019.v5.i20.1473

Abstract

Background: Osteosynthesis and partial patellectomy with patellar tendon repair are accepted procedures for inferior pole fracture of patella. Fractures of lower end of patella are usually avulsion injuries due to forceful flexion of knee against contracted quadriceps muscle. Multiple small fragments attached to patellar tendon make the management difficult. We conducted a prospective study of clinical and radiological outcome of inferior pole of patellar fractures treated with patellar tendon repair with non absorbable Ethibond and circumferential wiring. We compared the results with those of mid patellar fractures treated with modified tension band wiring technique.

Materials: We included all patients with lower pole patella fractures, presented to our department from 2012 to 2015. We included patients with fracture 34 A1 (b) (extra articular lower pole) and 34C1.3 (complete articular distal $3^{\text {rd }}$ fracture). These fractures were fixed with Ethibond 5 by making transosseous longitudinal drill holes and supplemented with circumferential stainless steel wires. Bostman scoring system was used to analyze and assess clinical results. We compared the results with midpatellar fractures treated by tension band wiring at the end of follow up period.

Results: We recruited 21 patients with inferior pole patella fractures. Independent $T$ Test was used to compare the means of Bostman scoring, flexion and loss of extension of knee joint between two groups of patients. Anterior knee pain and implant loosening was found more prevalent in midpatellar fracture treated by tension band wiring technique and required implant removal. There was no significant difference between two groups of patients.

Conclusion: Lower pole patella fracture can be successfully treated by this novel technique of fixation with less number of complications. Comminuted lower pole fractures which otherwise needed excision may be preserved with this technique.

Keywords: Inferior pole patella fracture, Osteosynthesis, circumferential wiring, Bostman scoring

\section{Introduction}

Patella plays a significant role in knee motion. Patellar fractures contribute to $1 \%$ of fractures. Proper understanding of extensor mechanism of knee joint has made preservation of lower pole of patella a necessity. It constitutes around 9 to $22 \%$ of operated patella fractures. Approximately $5 \%$ of patellar fractures involve inferior pole ${ }^{[1,2]}$. Patellar fractures are treated by tension band wiring technique a gold standard technique but comminuted fracture of lower pole of patella pose a challenge for every orthopaedician. Different treatment options like basket plating, separate vertical wiring ${ }^{[3]}$, wiring through cannulated screws, tension band wiring, partial Patellectomy are used by different surgeons. Circumferential wiring is an affective technique which may be used alone or with other modes of fixations ${ }^{[6]}$. Osteosynthes and partial patellectomy with patellar tendon repair are accepted procedures for inferior pole fracture of patella. The main objective of patellar fracture treatment is perfect reduction, maintaining extensor lever arm and attaining good range of movement across knee joint. Stable fractures with less than $2 \mathrm{~mm}$ of displacements may be treated conservatively provided there is no displacement at 60 degrees of flexion tested under $c$ arm guidance ${ }^{[12]}$.

Patella gives lever arm around knee joint. Fractures of lower end of patella are usually avulsion injuries due to forceful flexion of knee against contracted quadriceps muscle. Multiple small fragments attached to patellar tendon make the management difficult. 
Treating a patellar fracture with non absorbable suture materials yields equally good result as compared to metallic implants like $\mathrm{k}$ wire and stainless steel wires ${ }^{[5]}$.

The most important factor behind fracture treatment is early rehabilitation to avoid contractures of knee joint capsule and cartilage degeneration. Maintaining integrity of extensor mechanism and early mobilization is the main aim of treatment. Lower pole patella fractures are difficult to manage as they are multifragmentary in many cases. Recent studies have indicated that fiber wire and Ethibond are similar in strength to stainless steel wire ${ }^{[7,}{ }^{8]}$. We conducted a prospective study of clinical and radiological outcome of inferior pole of patellar fractures treated with patellar tendon repair with non absorbable Ethibond and circumferential wiring. In our knowledge this is the first study regarding this type of fixation. We compared the results with similar cohort of patients with mid patellar fractures treated with modified tension band wiring technique. We presumed that there is no difference in results of these two groups of patients. We assessed patients using Bostman scoring system.

\section{Materials and Methods}

The study was conducted in our hospital from 2012 to 2015. Ethical committee approval was obtained. We conducted a prospective comparative study recruiting all eligible candidates after obtaining their consent.

We included all patients with lower pole patella fractures, presented to our department from 2012 to 2015 . We excluded open fractures, patients with other bone fractures in the same limb and stiff knee. We included patients with extra articular lower pole and complete articular distal third fractures. A patient under general or spinal anesthesia under tourniquet, midline incision was put over knee joint. Denuded free bony fragments were removed but most of the bone stock was kept intact. Vertical drilling was done through patella from anterosuperior surface downwards. Ethibond no 5 stitches were taken through patellar tendon in Krackow fashion and then through distal pole fragments. Kwire was used to make small holes in distal patellar fragments through which Ethibond were passed using needle. These sutures were taken through vertical drill holes in main body of patella. These are tied over superior pole of patella. A $1.5 \mathrm{~mm}$ SS wire was used as circumferential encerclage. It was passed transversely in the mass of patellar tendon and taken around lateral border of patella, quadriceps muscle and then tightened and buried over superolateral pole. Vicryl no 1 was used to repair patellar retinacular ligaments. Different steps of surgery are shown in figures 1,2 and 3 .

Static quadriceps and ankle stretching exercises started from day one. These patients were followed regularly by 3,6 weeks. Long knee braces were used for 6 weeks. Knee range of movements started by $3^{\text {rd }}$ week. Partial weight bearing mobilization with long knee brace was started from $3^{\text {rd }}$ week onwards. Full weight bearing was started at the end of 6 weeks. Clinical and radiological assessments done every month until 3 month then at 6 th months and one year. $X$ rays of a patient are projected in figures 4,5 and 6 . We collected a cohort of displaced midpatellar fractures treated with modified tension band technique. Results of both groups were compared at the end.

We recruited a comparable cohort of mid patellar fractures selected by same surgeons. These were treated by open reduction and internal fixation with modified tension band wiring technique. Immobilization was continued for 6 weeks. Knee mobilization was started after plaster removal. Weight bearing was allowed. Patients were followed up in the department. Fracture union and range of movement was assessed. Bostman scoring system was used to analyze and assess clinical results. We noted down complications like infection, anterior knee pain, implant irritation and resurgery.

\subsection{Statistical analysis}

Data was entered in Microsoft excel (Windows 7; version 2007) and analysis were done in statistical package for social science (SPSS) version 16.0 for windows software. Level of significance was set as .05. Independent $\mathrm{T}$ Test was used to compare the means of Bostman scoring, flexion and loss of extension of knee joint between two groups of patients. Descriptive statistics with cross tables was done to assess the complications among these two groups.

\section{Results}

We studied 21 patients with inferior pole of fracture patella treated by this new technique. History of injury was collected. 10 patients sustained patella fracture due to slip and fall while 5 were while fall from height, remaining 6 during road traffic accidents. There were 15 male and 6 female patients in this group. The complication rate is given table 1 . Anterior knee pain and implant loosening was found more prevalent in midpatellar fracture treated by tension band wiring technique and required implant removal. Bostman scoring, knee flexion and loss of extension were compared by unpaired $\mathrm{T}$ test and depicted in table 2 . There was no significant difference among these groups as revealed by $\mathrm{p}$ value $>0.05$.

Table 1: Complications among two groups of patients.

\begin{tabular}{|c|c|c|c|c|c|c|}
\hline & & \multicolumn{4}{|c|}{ Complication } & \multirow{2}{*}{ Total } \\
\hline \multirow{2}{*}{ Cases } & & nil & Infection & implant loosening & anterior knee pain & \\
\cline { 2 - 7 } & inferior pole fracture & 16 & 2 & 3 & 0 & 21 \\
\cline { 2 - 7 } & mid patellar fracture & 9 & 2 & 6 & 4 & 21 \\
\hline \multicolumn{2}{|c|}{ Total } & 29 & 3 & 6 & 4 & 42 \\
\hline
\end{tabular}

Table 2: Bostman scoring, flexion and loss of extension (measured in degrees) compared between two groups.

\begin{tabular}{|c|c|c|c|c|c|c|}
\hline & Cases & $\mathbf{N}$ & Mean & Std. Deviation & Std. Error Mean & P value \\
\hline \multirow{2}{*}{ BOSTMAN } & inferior pole fracture & 21 & 27.29 & 2.125 & .464 & \\
\cline { 2 - 7 } & mid patellar fracture & 21 & 26.38 & 2.061 & .450 & .169 \\
\hline \multirow{2}{*}{ Flexion } & inferior pole fracture & 21 & 117.62 & 8.309 & 1.813 & .108 \\
\cline { 2 - 7 } & mid patellar fracture & 21 & 113.33 & 8.563 & 1.869 & \\
\hline \multirow{2}{*}{$\begin{array}{c}\text { Extension } \\
\text { Loss }\end{array}$} & inferior pole fracture & 21 & 1.95 & 2.156 & .470 & .617 \\
\cline { 2 - 7 } & mid patellar fracture & 21 & 2.29 & 2.125 & .464 & \\
\hline
\end{tabular}




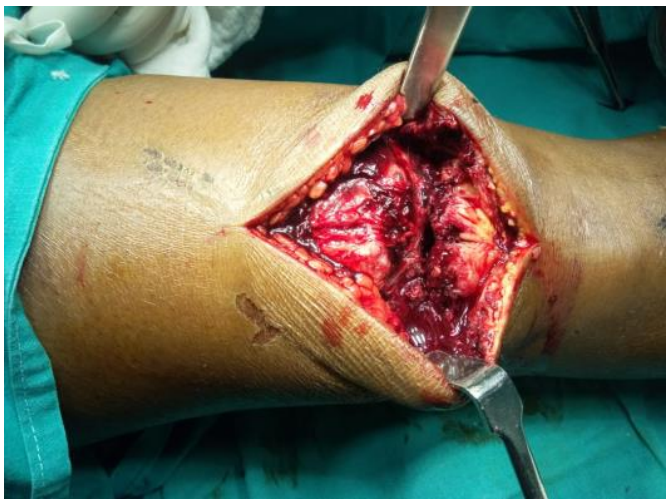

Fig 1: Intra operative picture showing separated fracture ends.

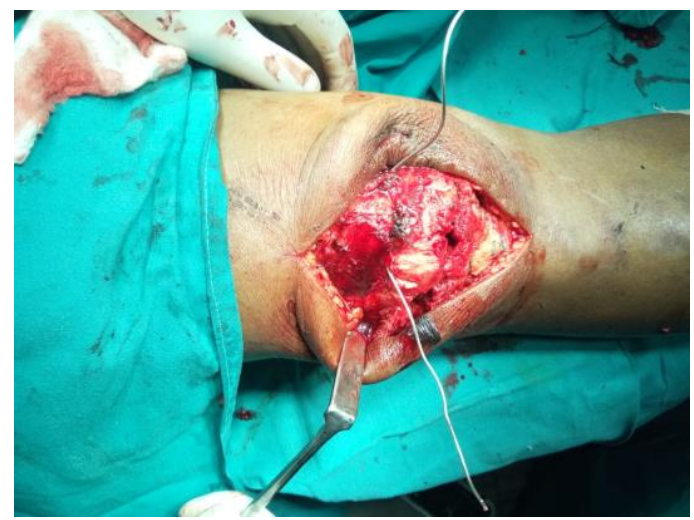

Fig 2: Fracture already fixed with Ethibond and stainless steel wire being introduced

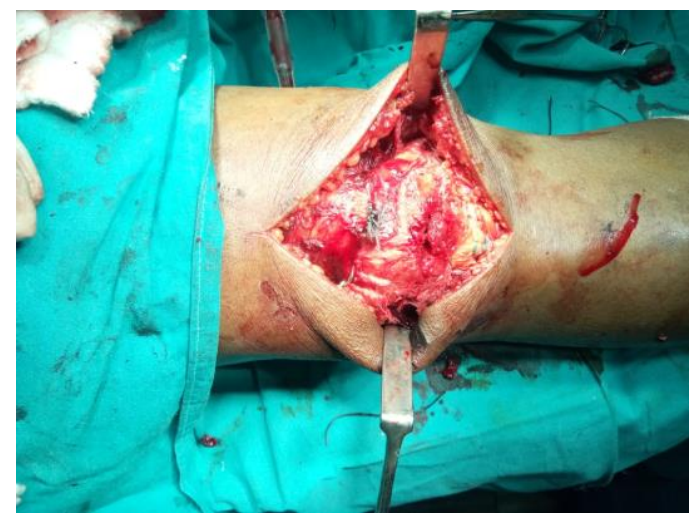

Fig 3: Fracture being fixed with Ethibond and circumferential wiring.

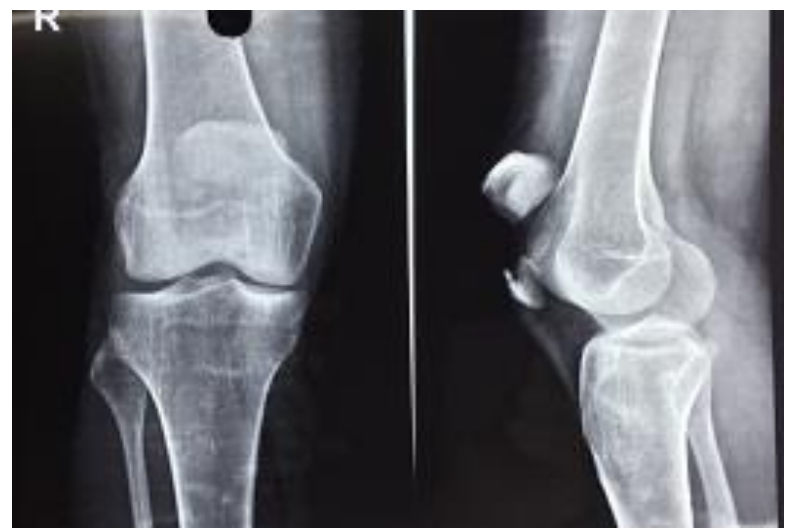

Fig 4: Preoperative $x$ ray of a lower pole patella fracture

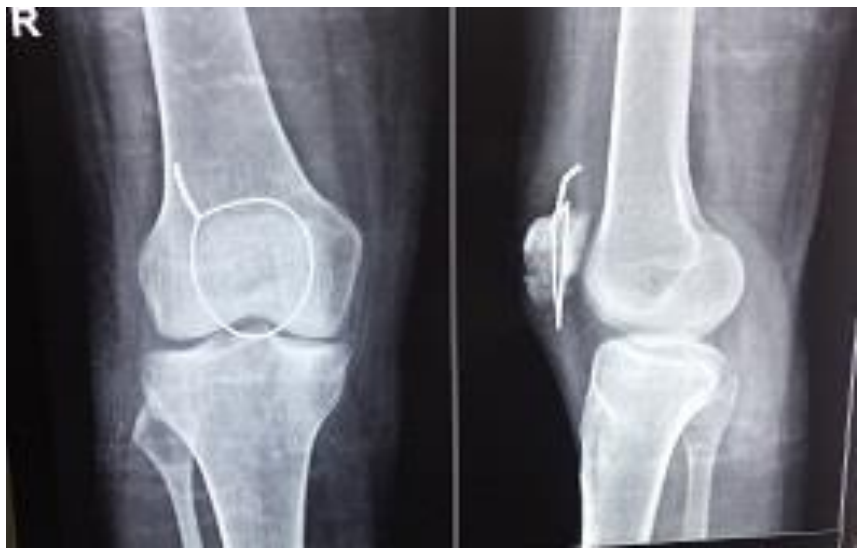

Fig 5: $\mathrm{X}$ ray picture at 3 months follow up

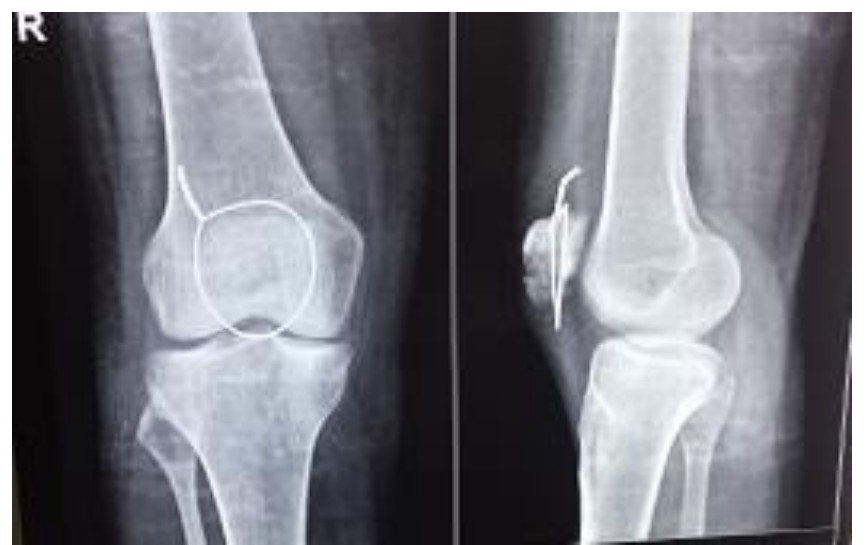

Fig 6: United fracture at one year follow up

\section{Discussion}

This study of functional assessment of inferior pole patella fractures was done as a prospective study in our institution. The assessment period was from 2012 to 2015. We noted range of movements, fracture union, and other complications like infection, second surgery. We used Ethibond as suture material which is found to be strong enough to steel wires by biomechanical studies in fixation of patella fractures ${ }^{[5]}$. Extra stability was provided by using circumferential stainless steel wire of size $1.5 \mathrm{~mm}$ for early rehabilitation and to avoid cut out chances over fracture site. The time for bony union was around 8 to 9 weeks.

In an article by Hung LK et al. studied partial patellectomy tension band wiring early mobilization. They reinforced the bone patellar tendon repair with figure of eight wiring attached superiorly to patella and inferiorly to tibia near tibial tuberosirly. Early mobilization was started and good result achieved by 3 months. Many patients had radiological osteoarthritis ${ }^{[9]}$. Our study has got similarities to this study but few differences. We tried to preserve maximum bone attached to patellar tendon and reinforced repair with circumferential wiring. Radiological osteoarthritis is relatively less in our cases.

Kenneth egol et al. did a comparative study of lower pole patella fracture treated with nonabsorbable suture to mid patellar fractures fixed with modified tension band wire. There was no difference at one year in two cohorts. Additionally there was no hard ware related complications in inferior pole repaired patients ${ }^{[10]}$. 
We added circumferential wiring as an additional support to repair which helped in early mobilization. These metallic wires were bent, folded and buried inside quadriceps muscle and did not cause skin irritation and other complications similar to $\mathrm{k}$ wire.

Veselko et al. compared inferior pole patella excision and repair to Osteosynthesis with basket plate. The final evaluation was based on Patellofemoral score and measurement of patellar height radio graphically. There was significant difference in both groups concluding that Osteosynthesis had good outcome ${ }^{[11]}$.

In a retrospective comparative study of inferior pole patella fracture treatment with basket plate in comparison to partial patellectomy, Matejcic et al. found excellent results with former method. Basket plates resulted in good osseous consolidation and early mobilization. There was significant difference with respect to level of activity, knee pain, and range of motion, muscular atrophy, muscular strength and patellofemoral score ${ }^{[12]}$. In our part of country availability and cost of basket plate may be a constraint. Our study evaluated the results of simple technique with cheaper and easily available stainless steel wire along with monofilament suture which yielded good functional and radiographic results. A study conducted by Hyung Keun Song et al. did a clinical and biomechanical study comparing the results fixation by separate vertical wiring alone and combined technique of cerclage plus vertical wiring. They came out with the result which estimated the loads to failure for separate wiring and combined technique were $216.4 \pm 72.4 \mathrm{~N}$ and $324.9 \pm 50.6 \mathrm{~N}$, respectively. Their findings showed enough evidence to show that combined technique provided strong construct for protected early exercises of knee joint and successful healing ${ }^{[13]}$. New options like anchor suture fixations did not prove to be superior except for operational time in comparison to partial patellectomy.

This study combines the advantage of two fixation devices like braided suture material with circumferential steel wire. In our knowledge this is the first article presenting the results of circumferential wiring and suturing combination for inferior pole patella fractures. This provided a stable construct to start knee mobilization and weight bearing. This prospective study helped us to investigate the time for bony union in both group of patients. A long term study is required to know the patellofemoral osteoarthritis in these patients. A multicentre study with more patients is essential to substantiate benefits of this treatment method.

\section{Conclusion}

Lower pole patella fracture can be successfully treated by this novel technique of fixation with less number of complications. Comminuted lower pole fractures which otherwise needed excision may be preserved with this technique. The results are comparable to midpatellar fractures treated by modified tension band wiring.

\section{Acknowledgements}

I acknowledge all the patients who gave permission to participate in this study.

\section{Declarations}

7.1 Funding: Nil

7.2 Conflict of interest: Nil

7.3 Ethical approval: Approved

\section{References}

1. Cramer KE, Moed BR. Patellar Fractures: Contemporary Approach to Treatment. J Am Acad Orthop Surg. 1997; 5:323-31.

2. Harris RM. Fractures of the patella and injuries to the extensor mechanism. In: Rockwood CA, Green DP, Bucholz RW, editors. Rockwood and Green's Fractures in adults. 6th ed. Philadelphia: Lippincott Williams \& Wilkins, 2006, 1969-97.

3. Yang KH, Byun YS. Separate vertical wiring for the fixation of comminuted fractures of the inferior pole of the patella. J Bone Joint Surg Br. 2003; 85:1155-60.

4. Bostrom MP, Asnis SE, Ernberg JJ, Wright TM, Giddings VL, Berberian WS, et al. Fatigue testing of cerclage stainless steel wire fixation. J Orthop Trauma. 1994; 8(5):422-8.

5. Patel VR, Parks BG, Wang Y, Ebert FR, Jinnah RH. Fixation of patella fractures with braided polyester suture: a biomechanical study. Injury. 2000; 31:1-6.

6. Ong TK, Chee EK, Wong CL, Thevarajan K. Fixation of Comminuted Patellar Fracture with Combined Cerclage and Tension Band Wiring Technique. Malaysian Orthopaedic J. 2008; 2(2):40-42.

7. Najibi S, Banglmeier R, Matta J, Tannast M. Material properties of common suture materials in orthopaedic surgery. Iowa Orthop J. 2010; 30:84-8.

8. Wright PB, Kosmopoulos V, Cote RE, Tayag TJ, Nana AD. Fiber Wire is superior in strength to stainless steel wire for tension band fixation of transverse patellar fractures. Injury. 2009; 40:1200-3.

9. Hung LK, Lee SY, Leung KS, Chan KM, Nicholl LA. Partial patellectomy for patellar fracture: tension band wiring and early mobilization J Orthop Trauma. 1993; 7(3):252-60.

10. Kenneth Egol MD, Daniel Howard BS, Roy Davidovitch BS. Patella Fracture Fixation with Suture and Wire: you reap what you Sew Iowa Orthop J. 2014; 34:63-67.

11. Veselko M, Kastelec M. Inferior patellar pole avulsion fractures: Osteosynthesis compared with pole resection. Surgical technique. J Bone Joint Surg Am. 2005; 1(1):113-21.

12. Matejcic A, Puljiz Z, Elabjer E, Bekavac-Beslin M, Ledinsky M. Multifragment fracture of the patellar apex: basket plate Osteosynthesis compared with partial patellectomy. Arch Orthop Trauma Surg. 2008; 128(4):403-8.

13. Hyung Keun Song, Je Hyun Yoo, Kyu Hyun Yang. Separate Vertical Wiring for the Fixation of Comminuted Fractures of the Inferior Pole of the Patella. Yonsei Med J 2014; 55(3):785-791. 